\title{
Management of catheter-related bloodstream infection due to coagulase-negative staphylococci
}

\author{
KW Choi \\ Associate Consultant (Infectious Diseases), Department of Medicine, Alice Ho Miu Ling Nethersole Hospital, Hong Kong
}

TITLE Management of the catheter in documented catheter-related coagulasenegative staphylococcal bacteremia: remove or retain?

AUTHORS Raad I, Kassar R, Ghannam D et al.

JOURNAL Clin Infect Dis 2009; 49: I I87-94. doi:I0.1086/605694

DECLARATION OF INTERESTS No conflict of interests declared.

Published online December 2009

Correspondence to KW Choi, Department of Medicine, Alice Ho Miu Ling Nethersole Hospital, II Chuen On Road, Tai Po, Hong Kong

tel. +85226892260

e-mail choikwl@ha.org.hk

\section{SUMMARY}

In this study, Raad and co-workers attempted to determine the impact of the removal of the central venous catheters (CVC) on the management of catheterrelated bloodstream infection (CRBSI) due to coagulasenegative staphylococcus (CoNS). They retrospectively evaluated 188 episodes of CoNS CRBSI in a cancer centre at Houston, USA, during the period from July 2005 to December 2007. In addition to compatible clinical features and the fulfillment of definition criteria of the Infectious Diseases Society of America for probable or definite CRBSI,' diagnosis of CoNS CRBSI was established by the following three criteria:

I. At least two blood cultures from any source should be available.

2. At least one blood culture should yield a quantitative count of $\geq 15$ colony-forming units (CFU) $/ \mathrm{ml}$.

3. The blood samples that yielded positive culture results should have been collected within a 72-hour period.

Multivariate logistic regression was used to identify factors associated with the failure of resolution of bacteraemia and recurrence of bacteraemia during a four-month follow-up period.

Five out of the 188 patients developed complications (four cases of septic phlebitis of a great vein, one case of endocarditis). No significant differences were found between patients with and without complications. Bacteraemia did not resolve in 13 cases; these cases were associated with stay in the intensive care unit prior to infection (odds ratio [OR] 7.0; 95\% confidence interval [Cl] I.5-32.6) and presence of other current site(s) of infection (OR 3.8; 95\% Cl I.I-I3.3). The management of CVCs (removal vs retention) did not appear to affect the resolution of bacteraemia. In the 175 patients who achieved resolution of bacteraemia, recurrence occurred in 17 patients. They were associated with the retention of the CVC (OR 6.6; 95\% Cl I.8-23.9) and presence of an infusion port (OR I5.I; 95\% Cl 3.2-70.2).

\section{OPINION}

Coagulase-negative staphylococci are commensal skin organisms that often contaminate blood culture specimens. Once considered as clinically unimportant organisms with low virulence, they are increasingly recognised nowadays as an important cause of nosocomial bloodstream infection. ${ }^{2}$ One important factor that has contributed to the rise of this pathogen is the use of CVCs, and patients involved are often immunocompromised (e.g. patients in intensive care, recipients of chemotherapy for malignancy, patients on haemodialysis, etc.). The formation of microbial biofilms on the CVCs, which are not eradicated by conventional antimicrobial therapy, is the potential source of recurrent bacteraemia and related complications.

Catheter retention has been shown to be an acceptable strategy in the management of CoNS CRBSI, and this was adopted by international guidelines.' However, studies that supported this strategy often had the following limitations:

- The case definition was not strict, which might lead to the inclusion of cases with possible blood culture contaminations (particularly if the blood specimens were drawn from the CVC). ${ }^{3}$

- The resolution of bacteraemia and hence acute infection was used as the primary outcome measure and the issue of recurrence was left unaddressed.

The work by Raad and co-workers sheds light on these unresolved problems. They used a strict definition of CoNS CRBSI to avoid the problem of blood culture contamination. They demonstrated that while retention of CVC did not significantly affect the resolution of CRBSI, the risk of recurrent bacteraemia was substantial. In addition, they also showed that the presence of an infusion port further increased the risk of recurrence, and they offered the explanation for this observation that intimate contact between the needle puncture of the port and the superficial skin is a direct pathway for the organism to gain entry into the port.' 
So, what have we learnt from this study? Although limited by its retrospective and non-randomised nature, it reminds us of the importance of proper microbiological work-up for patients with suspected CRBSI, the risk of recurrent bacteraemia following catheter retention and how the catheter type matters. ${ }^{4,5}$

\section{REFERENCES}

I Mermel LA, Allon M, Bouza E et al. Guidelines for the management of intravascular catheter-related infections: 2009 update by the Infectious Diseases Society of America. Clin Infect Dis 2009; 49:I45. doi:10.1086/599376

2 Tokars Jl. Predictive value of blood cultures positive for coagulase negative staphylococci: implications for patient care and health care quality assurance. Clin Infect Dis 2004; 39:333-4I. doi:I0.1086/42I94I

3 Martinez JA, DesJardin JA, Aronoff $M$ et al. Clinical utility of blood cultures drawn from central venous or arterial catheters in critically ill surgical patients. Crit Care Med 2002; 30:7-13. doi:I0.1097/00003246-200201000-00002

4 Flynn PM, Willis B, Gaur AH, Shenep JL. Catheter design influences recurrence of catheter-related bloodstream infection in children with cancer. J Clin Oncol 2003; 21:3520-5. doi:10.1200/ CO.2003.03.0I2

5 Raad I, Davis S, Khan A et al. Impact of central venous catheter removal on the recurrence of catheter-related coagulase-negative staphylococcal bacteremia. Infect Control Hosp Epidemiol 1992; |3:2| 5-2|. doi: $10.1086 / 6465 \mid 2$

\section{Age-related macular degeneration is linked to cardiovascular disease}

TY Wong

Professor and Director, Singapore Eye Research Institute, Singapore National Eye Centre, Singapore, and Professor and Director, Retinal Vascular Imaging Centre, Centre for Eye Research Australia, University of Melbourne, Australia

TITLE Age-related macular degeneration and risk of coronary heart disease and stroke: the Cardiovascular Health Study

AUTHORS Sun C, Klein R, Wong TY

JOURNAL Ophthalmology 2009; I I6:1913-9. doi:I0.1016/j.ophtha.2009.03.046

DECLARATION OF INTERESTS No conflict of interests declared.
Published online December 2009

Correspondence to TY Wong, Singapore Eye Research Institute, II Third Hospital Avenue, Singapore 16875I

tel. +656322457 I e-mail ophwty@nus.edu.sg

\section{SUMMARY}

Age-related macular degeneration (AMD) is a leading cause of irreversible vision loss in elderly persons in the UK and many other countries.' Although the aetiology of AMD remains poorly understood, there is increasing evidence that AMD shares similar risk factors (e.g. smoking and hypertension) and common pathogenic mechanisms (e.g. inflammation and genetic factors) with cardiovascular diseases. ${ }^{2}$ However, there have been limited studies that have investigated whether AMD is a risk factor for cardiovascular disease, with somewhat inconsistent results to date..$^{3-5}$

The current study examines the association of AMD with the incidence of coronary heart disease (CHD) and stroke events using data from the Cardiovascular Health Study, a population-based cohort study of cardiovascular disease in adults 65 years of age and older, living in four communities in the USA. The baseline examination started in 1989, but the study population is derived from participants who were seen in 1997-98, when they had retinal photography to document the presence of AMD. Incident CHD and stroke events up to 2004 were ascertained using standardised methods.

Of the 1,786 persons free of CHD at the time of retinal photography, 303 developed incident CHD events over seven years. Participants with signs of early AMD $(n=277)$ had a higher cumulative incidence of CHD than those without early AMD ( $25.8 \%$ vs $18.9 \%, p=0.00 I)$. After controlling for age, gender, race, systolic and diastolic blood pressure, hypertension status, fasting glucose, triglyceride, low density lipoprotein cholesterol, cigarette smoking, pack years of smoking and C-reactive protein, the presence of early AMD was associated with an increased risk of incident CHD (hazard ratio [HR] I.57, 95\% confidence intervals $[\mathrm{Cl}] \mathrm{I} .17-2.22)$. Late 